\title{
suPAR is the circulating factor in some but not all FSGS
}

\section{Howard Trachtman and Jochen Reiser}

With the whole world focused on-one might say obsessed with - the World Cup, we find ourselves thinking about the role of referees in sport and medicine. The general consensus is that they are needed to decide whether a goal has been scored or whether a paper is valid. We would hope that most of the time they are not needed to decide whether a ball has cleared the goal line or whether a scientific finding is meaningful. Instead, we propose that referees are required to keep the ball in play or research moving forward rather than to adjudicate on what has happened.

These musings seem especially germane to comments made by Jeroen Deegens and Jack Wetzels regarding a preclinical study performed by Cathelin et al. ${ }^{1}$ that assessed the effect of soluble urokinase plasminogen activator receptor (suPAR) injection on proteinuria and glomerular structure (The search goes on: suPAR is not the elusive FSGS factor. Nat. Rev. Nephrol. 10, 431-432; 2014). ${ }^{2}$ Jochen Reiser has already published a comprehensive analysis of this study and the limitations inherent in extrapolating data from animal studies to human disease. ${ }^{3}$ Moreover, we do not want to comment again on the impact of isoforms of suPAR that are not detected using the currently available ELISA kits. ${ }^{4}$ Wetzels and his colleagues are conducting important studies into the role of suPAR in focal segmental glomerulosclerosis $(\text { FSGS })^{5}$ and their work is essential to understand if and how suPAR acts in this disease. However, we advise that definitive conclusions about the role of suPAR in FSGS and other glomerular diseases must be based on all of the published evidence and should not be made prematurely.

First, FSGS is a heterogeneous disorder. This fact is best illustrated by the wide spectrum of genetic mutations affecting various cellular compartments and organelles that lead to the characteristic histopathological lesions. ${ }^{6}$ Thus, suPAR is unlikely to be the pivotal circulating factor in all patients with a given type of glomerular disease. However, in some patients levels of suPAR correlate with the development of recurrent FSGS after renal transplantation, ${ }^{7}$ indicating that the molecule can contribute to glomerular barrier dysfunction and cause clinically relevant changes in kidney function. The effects of suPAR might vary between diseases and might be modulated by biological determinants such as autoantibody production or concomitant systemic infection. The challenge is to find the subgroups of patients in whom suPAR is an important circulating factor that contributes to proteinuria and glomerulosclerosis.

Second, suPAR might not only be involved in primary FSGS but might also contribute to glomerular barrier dysfunction in other prevalent kidney disorders. Two reports have documented elevated serum suPAR levels that correlate with the severity of kidney injury in patients with diabetic nephropathy. ${ }^{8,9}$ suPAR has also been implicated in the glomerular involvement induced by various infections including the Hantaan virus. ${ }^{10}$

Third, suPAR has been shown to trigger separate and distinct pathways of podocyte dysfunction-a migratory phenotype and podocyte apoptosis-depending on the cell microenvironment. Depending on the podocyte level of expression of acid sphingomyelinase-like phosphodiesterase $3 b$, suPAR either activates pathological integrin $\alpha v \beta_{3}$ or transforming protein RhoA activity. ${ }^{9}$ These findings suggest that suPAR is active in the glomerulus and potentially contributes to an abnormal phenotype in a wide range of glomerular diseases.

Finally, the report that suPAR levels are positively associated with an increased risk of cardiovascular death in patients with chronic kidney disease suggests that the molecule is involved in the cardiorenal syndrome. ${ }^{11}$ Based on these data alone, suPAR is an important molecule that requires investigation by nephrologists and scientists.

Thus, suPAR remains in play and possesses all of the requirements to be described as a circulating factor. We look forward to continued activity in this area and anticipate that figuring out where and how suPAR acts is likely to score goals for patients with FSGS and other glomerular diseases. Medical Center, 1735 West Harrison Street,
Cohn Building, Suite 724, Chicago, IL 60612 , USA (J.R.). NYU Langone Medical Center, CTSI, 227 East 30 ${ }^{\text {th }}$ Street, Room 3110, New York, NY 10016, USA (H.T.).

Correspondence to: H.T.

howard.trachtman@nyumc.org

\section{Acknowledgements}

J.R.'s work is supported by R01 grants DK089394, DK073495, and DK101350. H.T.'s work is supported in part by R01 grant DK100307.

\section{Competing interests}

H.T. declares no competing interests. J.R. is an inventor on pending and issued patents on SUPAR and its modification in renal disease.

1. Cathelin, D. et al. Administration of recombinant soluble urokinase receptor per se is not sufficient to induce podocyte alterations and proteinuria in mice. J.Am. Soc. Nephrol. 25, 1662-1668 (2014).

2. Deegens, J. K. \& Wetzels, J. F. The search goes on: suPAR is not the elusive FSGS factor. Nat. Rev. Nephrol. 10, 431-432 (2014).

3. Reiser, J. \& Chapman, H. Soluble urokinase-type plasminogen activator receptor in FSGS: stirred but not shaken. J. Am. Soc. Nephrol. 25, 1611-1613 (2014).

4. Schlöndorff, D. Are serum suPAR determinations by current ELISA methodology reliable diagnostic biomarkers for FSGS? Kidney Int. 85, 499-501 (2014).

5. Maas, R., Wetzels, J. \& Deegens, J. Serum suPAR concentrations in patients with focal segmental glomerulosclerosis with end-stage renal disease. Kidney Int. 85, 711 (2014).

6. D'Agati, V. Pathobiology of focal segmental glomerulosclerosis: new developments. Curr. Opin. Nephrol. Hypertens. 21, 243-250 (2012).

7. Alachkar, N. et al. Podocyte effacement closely links to suPAR levels at time of posttransplantation focal segmental glomerulosclerosis occurrence and improves with therapy. Transplantation 96, 649-656 (2013).

8. Theilade, S. et al. Soluble urokinase plasminogen activator receptor levels are elevated and associated with complications in patients with type 1 diabetes. J. Intern. Med. http://dx.doi.org/10.1111/joim.12269.

9. Yoo, T. et al. Sphingomyelinase-like phosphodiesterase $3 \mathrm{~b}$ expression levels determine podocyte injury phenotypes in glomerular disease. J. Am. Soc. Nephrol. http:// dx.doi.org/10.1681/ASN.2013111213.

10. Outinen, T. et al. Urine soluble urokinase-type plasminogen activator receptor levels correlate with proteinuria in Puumala hantavirus infection. J. Intern. Med. http://dx.doi.org/10.1111/ joim.12257.

11. Meijers, B. et al. Soluble urokinase receptor is a biomarker of cardiovascular disease in chronic kidney disease. Kidney Int. http:// dx.doi.org/10.1038/ki.2014.197. 